\title{
Scaling Properties of Optokinetic Nystagmus Amplitude Sequence
}

\section{Torbjørn Aasen ${ }^{1,2}$}

${ }^{1}$ Norwegian National Advisory Unit on Vestibular Disorders, Haukeland University Hospital, Bergen, Norway;

${ }^{2}$ Department of Otorhinolaryngology, Head and Neck Surgery, Haukeland University Hospital, Bergen, Norway

Correspondence to: Torbjørn Aasen, torbjorn@aasens.net

Keywords: Nystagmus, Optokinetic, Hurst Exponent, Information Processing

Received: October 5, $2020 \quad$ Accepted: November 13, $2020 \quad$ Published: November 16, 2020

Copyright $\odot 2020$ by author(s) and Scientific Research Publishing Inc.

This work is licensed under the Creative Commons Attribution International License (CC BY 4.0).

http://creativecommons.org/licenses/by/4.0/

\section{(c) (i) Open Access}

\section{ABSTRACT}

Optokinetic nystagmus (OKN) is rhythmic eye movements, back and forth, with a slow and fast phase when the eyes are presented for full-field visual stimulus. OKN was recorded in a healthy subject for four conditions, stripes moving $30 \% \mathrm{~s} \mathrm{left}$ and right and $60 \% \mathrm{~s} \mathrm{left} \mathrm{and}$ right. In this paper, surrogate data analysis was applied to test the Hurst exponents for increasing time horizons for the integrated OKN amplitude sequences statistically against 100 shuffled sequences (i.e., the serial dependency of the original data sequences is broken by changing the order of the sequence). The result shows that the pattern of the OKN amplitude sequence scales statistically different $(p<0.01)$ compared to random permutations of the same numbers for scaling shorter than 16 nystagmus components (slow and fast phases) for all four test conditions.

\section{INTRODUCTION}

When looking for identifiers for reduced functionality of a physiological system, we need to distinguish information pattern from random variations. The aim of this study was to investigate the possible presence of information pattern in, and the memory length of, the OKN amplitude sequence. Surrogate data analysis-using the Hurst exponent for increasing scaling length, as the statistical parameter-was applied to determine for how long, in terms of nystagmus phases, the statistical analysis showed significant differences between the integrated OKN amplitude sequence compared to the shuffled data, at 1\% level.

\subsection{Optokinetic Nystagmus}

When presented with a moving image, the eyes respond with a movement in the same direction as the image, interrupted by quick resetting phases [1-3]. These reflexive, rhythmic eye movements, which are called optokinetic nystagmus (OKN), interact with the vestibulo-ocular reflex and the smooth pursuit 
function to hold objects steady on the retina.

The optokinetic nystagmus amplitude (OKNA) sequence is the subsequent slow and fast phases of OKN amplitudes.

\subsection{Hurst Exponent}

The scaling coefficient $H$, the Hurst exponent, is a measure of memory of a time series with the three properties [4-8].

A time-integrated white noise time series (i.e., a random walk series) has a Hurst exponent of $H=0.5$.

A Hurst exponent that is higher than $0.5(H>0.5)$ corresponds to a process which is trending (persistent), also known as positive autocorrelation.

A Hurst exponent that is lower than $0.5(H<0.5)$ corresponds to a mean reverting process (anti-persistent) (i.e., negative autocorrelation).

\subsection{Surrogate Data Testing}

Surrogate data testing is a statistical technique that can be used to test if a sequence of data differs significantly from a random sequence; for example, a random permutation of the same data/numbers. New data sequences are generated from the original sequence by shuffling, randomly changing the order of the sequence (the same numbers but where the serial dependency is broken $[9,10]$ ). A parameter is calculated from the original data sequence and tested statistically against the distribution of the parameters from the shuffled sequences. The null hypothesis $H_{0}$ : Data ${ }_{\text {original }}=$ mean $^{\text {Data }}$ surrogates $_{\text {, }}$ that the original data sequence belongs to the same distribution as the surrogates can then be rejected at, for example, $1 \%$ probability level.

\section{MATERIAL AND METHODS}

\subsection{Subject}

$\mathrm{OKN}$ was recorded from a healthy subject for four conditions: stripes moving $30 \% \mathrm{~s} \mathrm{left}$ and right and $60 \%$ left and right.

\subsection{Recording Technique}

Horizontal eye movements were recorded with two electrodes (Ag-AgCl skin electrodes), which were placed lateral to each eye, along with a reference electrode at the center of the forehead. The signal was amplified (10 s time constant and an upper cut-off frequency of $30 \mathrm{~Hz}$ ) and digitized into a computer using $12 \mathrm{bit} \mathrm{A} / \mathrm{D}$ resolution and $100 \mathrm{~Hz}$ sampling frequency (sampling time $\tau \mathrm{s}=0.01$ ).

\subsection{Optokinetic Stimulation and Registration}

OKN was obtained by stimulating the visual field with $3.75^{\circ}$ width vertical light stripes separated by $11.25^{\circ}$ width dark stripes. A slit projector presented the stripes on the inside of a hemispherical screen $(100 \mathrm{~cm}$ in diameter). The subjects were sitting in front of a screen in a darkened room with the head restrained. The subjects were instructed to not follow the stripes with the eyes but to focus their vision on the screen, allowing the optokinetic reflex to control the eye movements. Recordings were performed with the movement of the stripes at a velocity of $30 \%$ and at $60 \%$, which are below and above the normal threshold for smooth pursuit function [11, 12]. Each recording lasted for $1 \mathrm{~min}$. Figure 1 shows a $1 \mathrm{~s}$ recording of $\mathrm{OKN}$.

\subsection{Methods and Analysis}

Analyzing methods for distinguishing random behavior from long-term correlation/memory in time series have been described (Mandelbrot and Van Ness [13]). It has been shown that the mean square 


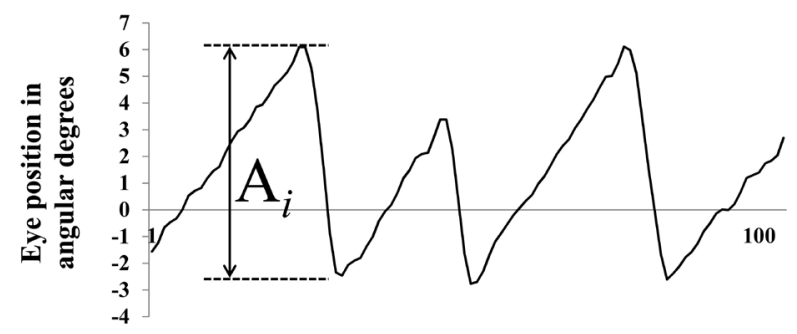

Figure 1. A $1 \mathrm{~s}$ registration of an optokinetic time series. Upward direction represents eye movement to the right and downward direction represents eye movement to the left.

displacement exhibit scaling laws proportional to $\Delta t^{2 H} \quad[14]$.

$$
\left\langle\Delta x^{2}\right\rangle=\left\langle\left(x_{i}-x_{i-\Delta t}\right)^{2}\right\rangle \sim \Delta t^{2 H}
$$

$\left\langle\Delta x^{2}\right\rangle$ is the mean square displacement, $\Delta t$ is the time interval and $H$ is the Hurst exponent. If data are independent, i.e. no memory, the displacement will increase with the square root of time and $H=$ $1 / 2$.

\subsection{The Algorithm}

The algorithm was applied to the integrated-the cumulative sum-of the nystagmus amplitude sequence series, $A_{p}$ adjusted for the mean.

$$
\{x\}_{i=1}^{n}=\sum_{i=1}^{n}\left(A_{i}-\text { mean } A_{i}\right)
$$

where

$$
\text { mean } A_{i}=\sum_{i=1}^{n} \frac{A_{i}}{n}
$$

The time series, $x_{\dot{p}}$ represents the integrated OKNA sequence. phases.

First, we calculated the mean square displacement $\left\langle\Delta x^{2}\right\rangle$ in measure of number of nystagmus

$$
\left\langle\Delta x_{i}^{2}\right\rangle_{k}=\left\{\frac{\sum_{i=1}^{-k+n}\left(x_{i+k}-x_{i}\right)^{2}}{-k+n}\right\}_{k=1}^{\beta=54}
$$

Then, from the scaling properties, we found the slope, $s_{i}$.

$$
s_{i}=\frac{1}{2} \frac{\log \left(\left\langle\Delta x_{i}^{2}\right\rangle_{k}\right)}{\log (k)}
$$

The scaling, the Hurst exponents for 50 time scales, was then calculated using the method of least square to fit straight lines for 1 to $n(n=4$ to 54$)$ nystagmus phases.

\subsection{Statistical Hypothesis Testing - Surrogate Data Analysis}

The Hurst exponents for increasing time horizons for the integrated original OKNA sequences were statistically tested against 100 shuffled sequences (i.e., the serial dependency of the original data sequences is broken by changing the order of the sequence) $[9,10]$.

The statistical $Z$ score is

$$
Z=\frac{H_{\text {orig }}-\text { mean } H_{\text {surr }}}{\operatorname{SD~} H_{\text {surr }}}
$$


where $H_{\text {orig }}$ is the Hurst exponent of the original integrated OKNA sequence, mean $H_{\text {surr }}$ is the mean value for the 100 surrogates

$$
\text { mean } H_{\text {surr }}=\sum_{i=1}^{n} \frac{H_{\text {surri }}}{n}
$$

and SD $H_{\text {surr }}$ is the standard deviation of the distributed Hurst exponents from the 100 surrogate data sets.

$$
\text { SD } H_{\text {surr }}=\sqrt{\frac{\left(H-\text { mean } H_{\text {surr }}\right)^{2}}{n}}
$$

\section{RESULTS}

The Hurst exponent parameters for the 50 time scales, $H_{\text {orig, }}$ were tested against the 50 time scales of the 100 shuffled surrogates. The null hypothesis H0: $H_{\text {orig }}=$ mean $H_{\text {surr }}$ is rejected when $Z>2.576$, which states that $H_{\text {orig }}$ is outside the $99 \%$ confidence interval of the shuffled distribution $(p<0.01)$ (Figure 2).

Surrogate data analysis shows that the pattern of the OKNA sequence analyzed in this study is statistically different $(p<0.01)$ compared to the random permutations of the same numbers for scaling shorter than 16 nystagmus phases for left $30^{\circ} / \mathrm{s},<53$ phases for right $30^{\circ} / \mathrm{s},<38$ phases for left $60 \%$ and $<35$ phases for right $60 \%$.

\section{DISCUSSION}

Earlier studies $[3,15]$ have discussed the inter-relation between the fast and slow phases of nystagmus. One study found a lower correlation between the amplitude of the slow phase and the following fast phase compared to the correlation between the fast phase and the following slow phase, which was interpreted as the eye is not determined by the previous slow phase but is free to move voluntarily in order to focus on an object of interest [15]. This study has focused on the sequence of following slow and fast phases and found that the physiological regulating system keeps memory beyond subsequences of nystagmus beats. The pattern of information and memory is essential when looking for identifiers for reduced functionality of a physiological system.

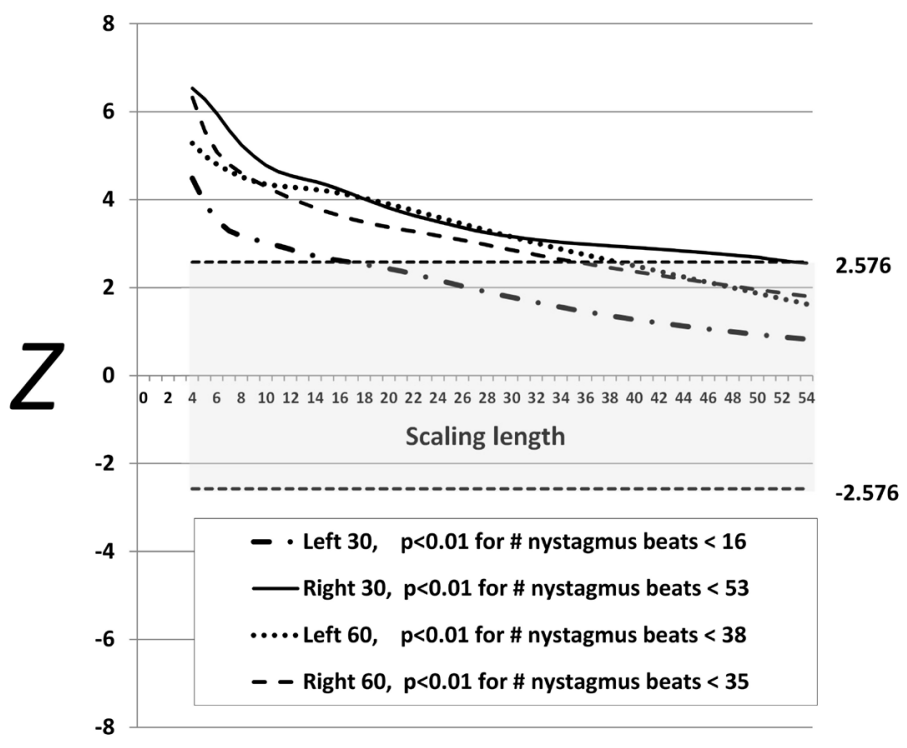

Figure 2. The Hurst exponent parameter, Horig, tested against 100 shuffled surrogates. The null hypothesis $H_{0}: H_{\text {orig }}=$ mean Hsurr is rejected when $Z>2.576$, which states that $H$ is outside the $99 \%$ confidence interval of the shuffled distribution $(p<0.01)$. 


\section{CONCLUSION}

Testing the displacement of the integrated OKNA sequences from passive (subcortical) stare full-field OKN stimulation for four conditions, stripes moving $30 \% \mathrm{~s} \mathrm{left} \mathrm{and} \mathrm{right} \mathrm{and} 60 \% \mathrm{~s} \mathrm{left} \mathrm{and} \mathrm{right} \mathrm{from} \mathrm{a}$ healthy subject as a function of time span in measure of number of nystagmus beats/phases, shows that the amplitude of the fast and slow nystagmus phases scales statistically different $(p=0.01)$ compared to random permutations of the same numbers for scaling shorter than 16 nystagmus components for all four test conditions. It will be interesting to see if this will be affected for conditions related to vertigo symptoms.

\section{CONFLICTS OF INTEREST}

The author declares no conflicts of interest regarding the publication of this paper.

\section{REFERENCES}

1. Magnusson, M., Schalén, L., Pyykkö, I., Enbom, H. and Henriksson, N.G. (1988) Clinical Considerations concerning Horizontal Optokinetic Nystagmus. Acta Oto-Laryngologica. Supplementum, 455, 53-57. https://doi.org/10.3109/00016488809125057

2. Norouzifard, M., Black, J., Thompson, B., Klette, R. and Turuwhenua, J. (2020) A Real-Time Eye Tracking Method for Detecting Optokinetic Nystagmus. In: Palaiahnakote, S., Sanniti di Baja, G., Wang, L., Yan, W., Eds., Pattern Recognition, ACPR 2019, Lecture Notes in Computer Science, vol 12047, Springer, Cham. https://doi.org/10.1007/978-3-030-41299-9_12

3. Chun, K.S. and Robinson, D.A. (1978) A Model of Quick Phase Generation in the Vestibuloocular Reflex. Biological Cybernetics, 28, 209-221. https://doi.org/10.1007/BF00344268

4. Collins, J.J. and De Luca, C.J. (1994) Random Walking during Quiet Standing. Physical Review Letters, 73, 764-767. https://doi.org/10.1103/PhysRevLett.73.764

5. Palatinus, Z., Dixon, J.A. and Kelty-Stephen, D.G. (2013) Fractal Fluctuations in Quiet Standing Predict the Use of Mechanical Information for Haptic Perception. Annals of Biomedical Engineering, 41, 1625-1634. https://doi.org/10.1007/s10439-012-0706-1

6. Gorshkov, O. and Ombao, H. (2019) Evaluation of Monofractal and Multifractal Properties of Inter-Beat (R-R) Intervals in Cardiac Signals for Differentiation between the Normal and Pathology Classes. IET Signal Processing, 13, 798-805. https://doi.org/10.1049/iet-spr.2018.5536

7. Chen, Y.C., Lin, L.L, Lin, Y.T, Hu, C.L. and Hwang, I.S. (2017) Variations in Static Force Control and Motor Unit Behavior with Error Amplification Feedback in the Elderly. Frontiers in Human Neuroscience, 11, 538. https://doi.org/10.3389/fnhum.2017.00538

8. Goel1, R., De Dios, Y.E., Gadd, N.E., Caldwell, E.E., Peters, B.T., Reschke, M.F., Bloomberg, J.J, Oddsson, L.I.J. and Mulavara, A.P. (2017) Assessing Somatosensory Utilization during Unipedal Postural Control. Frontiers in Systems Neuroscience, 11, 21. https://doi.org/10.3389/fnsys.2017.00021

9. Shelhamer, M. (1997) On the Correlation Dimension of Optokinetic Nystagmus Eye Movements: Computational Parameters, Filtering, Nonstationarity, and Surrogate Data. Biological Cybernetics, 76, 237-250. https://doi.org/10.1007/s004220050336

10. Aasen, T., Nordahl, S.H.G., Goplen, F.K. and Knapstad, M.K. (2019) The Turning Point for Maintaining Balance. Journal of Diagnostic Techniques and Biomedical Analysis, 8, 1.

11. Schalén, L. (1980) Quantification of Tracking Eye Movements in Normal Subjects. Acta Oto-Laryngologica, 90, 404-413. https://doi.org/10.3109/00016488009131742

12. Spalton, D.J. (1984) Neuro-Ophthalmology. In: Spalton, D.J., Hitchings, R.A. and Hunter, P.A., Eds., Slide Atlas 
of Ophthalmology, Gower Medical Publishing Ltd., London, 19, 505.

13. Mandelbrot, B.B. and van Ness, J.W. (1968) Fractional Brownian Motions, Fractional Noises and Applications. SIAM Review, 10, 422-437. https://doi.org/10.1137/1010093

14. Feder, J. (1988) Fractals. Plenum, New York, NY. https://doi.org/10.1007/978-1-4899-2124-6

15. Aasen, T., Goplen, F.K. and Nordahl, S.H.G. (2013) Short-Term Information Pattern in Optokinetic Nystagmus Amplitude Time Series. Journal of Vestibular Research, 23, 71-75. https://doi.org/10.3233/VES-130481 